Revue

Revue de l'histoire des religions

de Ihistoire des religions

1 | 2017

Varia

\title{
Érasme dans l'œuvre critique de Richard Simon
}

Erasmus in the Critical Works of Richard Simon

Jacques Le Brun

(2) OpenEdition

Journals

Édition électronique

URL : http://journals.openedition.org/rhr/8655

DOI : $10.4000 /$ rhr.8655

ISSN : $2105-2573$

Éditeur

Armand Colin

Édition imprimée

Date de publication : 1 mars 2017

Pagination : 73-88

ISBN : 978-2-200-93125-4

ISSN : 0035-1423

\section{Référence électronique}

Jacques Le Brun, «Érasme dans l'œuvre critique de Richard Simon », Revue de l'histoire des religions

[En ligne], 1 | 2017, mis en ligne le 01 mars 2019, consulté le 06 janvier 2021. URL : http://

journals.openedition.org/rhr/8655; DOI : https://doi.org/10.4000/rhr.8655

Tous droits réservés 


\section{Érasme dans l'œuvre critique de Richard Simon}

Érasme au XVI e siècle, Richard Simon au XVII apparaissent comme les figures marquantes de la critique scripturaire à l'âge classique. Richard Simon n'a cessé de s'interroger sur les pratiques, les principes et la théologie d'Érasme; d'un côté il était sévère envers le travail d'un traducteur qui ignorait l'hébreu et les principes de la critique, de l'autre il admirait en Érasme un lettré bon connaisseur de l'Antiquité et un homme qui avait tenté une entreprise de traduction et de paraphrase du Nouveau Testament trop haute pour lui ; Simon, comme ses contemporains, relevait surtout chez Érasme, outre la hardiesse à combattre les abus, les constantes réserves à l'égard de saint Augustin et de l'augustinisme, position théologique que l'ancien oratorien ne cessera de tenir.

\section{Erasmus in the Critical Works of Richard Simon}

Erasmus in the $16^{\text {th }}$ Cand Richard Simon in the $17^{\text {th }}$ stand out as remarkable figures of scriptural criticism in the Classical period. Richard Simon never ceased to call into question Erasmus's practices, principles and theology; on the one hand, he looked severely on the work of a translator who was not versed in Hebrew or in the pricinciples of criticism; on the other, he admired Erasmus as a man of letters with extensive knowledge of Antiquity and as one who had attempted the over-ambitious task of translating and paraphrasing the New Testament. Simon, like his contemporaries, in addition to Erasmus's boldness in combatting abuses, especially noted his constant reservations regarding Saint Augustine and Augustinism, a theological position that the former Oratorian always maintained. 
Henri de Lubac affirmait naguère d'Érasme : « On ne lui connaît pas de postérité définie. Son influence est demeurée diffuse $»^{1}$. Le jugement est particulièrement justifié dans les domaines de l'érudition patristique et de l'exégèse du Nouveau Testament: tout le monde utilise ses travaux, en reconnaît l'intérêt, mais chez personne cette influence n'apparaît comme centrale ou déterminante. Même si un Grotius le suit souvent de près, on ne peut dire qu'il est «disciple » d'Érasme. Même si Richard Simon pense sans cesse à lui lorsqu'il traduit et commente le Nouveau Testament et qu'il travaille à établir le texte de saint Jérôme, Érasme n'est pour lui ni un modèle, ni une référence exclusive.

\section{ÉRASMe AU MILIEU DU XVII ${ }^{\mathrm{e}}$ SIÈCLE}

Richard Simon en effet a lu Érasme dès ses années de formation à l'Oratoire, et il a réfléchi sur la méthode et la théologie érasmiennes ${ }^{2}$. Le premier témoignage que nous ayons date d'une époque où, encore oratorien, Simon participait à des discussions qui réunissaient à Paris, sans doute à l'hôtel de Liancourt, érudits, lettrés, beaux esprits et théologiens entre 1670 et $1671^{3}$. Le nom d'Érasme revenait souvent dans ces conversations, Érasme critique des «friponneries des moines débauchés de son temps » ${ }^{4}$, Érasme dont on appréciait les lettres ${ }^{5}$ et dont la vie avait été écrite par M. Joly, chanoine de Notre-Dame ${ }^{6}$. Coutelier résumait assez bien

1. Henri de Lubac, Exégèse médiévale, $\mathrm{II}^{\mathrm{e}}$ partie, t. 2, Paris, Aubier, 1964, p. 459. Sur "l'héritage érasmien» au XVII ${ }^{\mathrm{e}}$ siècle, voir François Laplanche, L'Écriture, le sacré et l'histoire. Érudits et politiques protestants devant la Bible en France au XVII siècle, Amsterdam et Maarssen, APA - Holland University Press, 1986, p. 41-45 et passim.

2. Sur Richard Simon, voir Paul Auvray, Richard Simon (1638-1712). Étude bio-bibliographique avec des textes inédits, Paris, P.U.F., 1974 ; et Jacques Le Brun, article «Simon (Richard) », Dictionnaire de la Bible, Supplément, fasc. 17, t. XII, Paris, Letouzey et Ané, 1996, col. 1353-1383.

3. Voir Jean Lesaulnier, Port-Royal insolite. Édition critique du Recueil de choses diverses, Paris, Klincksieck, 1992.

4. Selon Patru, ibid., p. 176.

5. Selon Manissier, ibid., p. 326, et voir aussi p. 562.

6. Vie dont la publication ne fut pas permise, voir B.N., ms. fr. 22582, p. 292. Voir aussi pour Claude Joly, Bibliothèque de l'Arsenal, ms. 4625. 
l'opinion générale en ce milieu : «Il n'était point théologien. [...] Il faut distinguer deux temps dans Érasme: dans sa jeunesse, il s'est tout appliqué aux belles-lettres, après à imprimer les Pères. Il ne paraît pas dans Érasme qu'il aille bien dans la piété. Ce n'est qu'une dévotion qui va à ses aises. Son style railleur n'est pas des Saints-Pères $»^{7}$.

Richard Simon, qui avait participé à plusieurs reprises à ces conversations en érudit prodige «fort savant dans les langues $»^{8}$, parlant de saint Augustin, de saint Jérôme, des Septante, des Pères grecs et du débat alors très actuel entre Jean Claude et Pierre Nicole $^{9}$, jugeait en ces termes la Paraphrase du Nouveau Testament par Érasme : «Il a détourné quelques passages de sa paraphrase à son pélagianisme, nestorianisme, à ses idées de Jésus-Christ sur sa descente aux enfers, sur le Purgatoire; elle est pourtant belle $»^{10}$, jugement dont nous retrouverons le caractère nuancé à plusieurs reprises sous la plume de Simon critique du Nouveau Testament.

Quel visage offrait alors le souvenir d'Érasme aux hommes du XVII siècle? C'est en rapport avec les jugements de ses contemporains que se comprend la position de Richard Simon. Pour ceux qu'on appelle les «libertins érudits », Érasme apparaissait comme un héros vénéré. Guy Patin jugeait les Épîtres un «trésor incomparable », «le meilleur livre de [son] étude » et possédait chez lui le portrait de l'humaniste ${ }^{11}$, Nicolas Bourbon trouvait en lui un maître qui l'initiait à la pensée ${ }^{12}$. Exprimant une opinion qui sera courante au milieu du XVII ${ }^{\mathrm{e}}$ siècle et que partageait Guy Patin, Grotius écrivait: «J'aime Érasme pour bien des raisons, dont la première est qu'il a été le commun maître et précepteur de tout le genre humain ; la seconde est qu'il a crié contre les abus qu'il a vus aux ecclésiastiques sans faire schisme et sans se séparer de l'Église. C'est comme devaient faire les hérésiarques du siècle passé, qui en faisant schisme ont causé tant de maux $»^{13}$.

7. Dans Jean Lesaulnier, Port-Royal insolite, p. 692-693.

8. Ibid., p. 391.

9. Ibid., p. 292, 374, 634, 673, 676-679.

10. Ibid., p. 693.

11. Henri Busson, La pensée religieuse française de Charron à Pascal, Paris, Vrin, 1933, p. 503 ; René Pintard, Le libertinage érudit dans la première moitié du XVII siècle, nouv. éd., Genève, Slatkine, 2000, p. 162, 262.

12. René Pintard, Le libertinage érudit, p. 314.

13. Cité ibid., p. 324. 
Qu'Érasme apparaisse comme le critique des abus, comme celui qui contestait l'authenticité de maintes reliques ${ }^{14}$, ne gênait nullement les plus dévots, tel le prédicateur Jean de Lingendes, évêque de Sarlat puis de Mâcon, sur qui Tallemant des Réaux raconte : «Cela me fait souvenir de M. de Mâcon, Lingendes qui disait que les trois livres qu'il aimait le mieux, c'était la Bible, Érasme et l'Astrée $»^{15}$. Néanmoins une ambiguïté demeurait toujours dans les jugements portés sur l'humaniste: avait-il été seulement un "grammairien, un savant», comme le définissait avec mépris le Charles-Quint d'un des Dialogues des morts modernes de Fontenelle en $1683^{16}$, même si dans le dialogue Érasme se défendait en soutenant que la science n'était pas acquise par succession mais était à la fois «difficile à recueillir » et «fort honorable $»^{17}$ ? Dans un livre posthume qui était un véritable plaidoyer apologétique en faveur d'Érasme, un ecclésiastique rigoriste, curé de Triel puis prieur de Beaulieu Saint-Avoye, Jean Richard, qui avait de vives sympathies port-royalistes et était soucieux de réforme pastorale ${ }^{18}$, exposait toutes les raisons d'admirer Érasme et répondait aux calomnies qui, déjà du vivant de l'humaniste, tentaient de le noircir. Richard notait ainsi qu' on le considérait ordinairement « comme un grammairien ou tout au plus comme un docteur illustre dans les belles-lettres $»^{19}$, mais c'était pour exalter «sa profonde érudition » que manifestaient ses Notes sur l'Évangile, ses Paraphrases du Nouveau Testament, ses éditions des Pères et ses traités de piété ${ }^{20}$ : Érasme aurait aussi été «le plus savant homme de son siècle $»^{21}$, dénoncé comme hérétique par l'ignorance et la barbarie et par « quelques moines mendiants » qui

14. Voir Henri Busson, La religion des classiques, Paris, P.U.F., 1948, p. 312, 392.

15. Tallemant des Réaux, Historiettes, t. II, Paris, Gallimard (Pléiade), 1960-1961, p. 324.

16. Fontenelle, Euvres diverses, t. I, La Haye, 1738, p. 36-37.

17. Ibid., p. 38.

18. Sur lui (1615-1686) et sur ses ouvrages, voir Jeanne Ferté, La vie religieuse dans les campagnes parisiennes (1622-1695), Paris, Vrin, 1962, p. 218, 274, 314 ; Jean Lesaulnier, Port-Royal insolite, op. cit., p. 798 et passim; et JeanLouis Quantin, Le catholicisme classique et les Pères de l'Église, Paris, Études Augustiniennes, 1999, p. 512-513 et passim.

19. [Jean Richard], Sentimens d'Érasme de Rotterdam, conformes à ceux de l'Église catholique sur tous les points controversés, Cologne, 1688, p. 1.

20. Ibid., p. 2-3.

21. Ibid., p. 7, 211. 
tiraient profit des abus ${ }^{22}$; ses critiques des «sophistiqueries que les docteurs enseignaient dans les Écoles $»^{23}$ alimentaient contre Érasme les calomnies, et sa dénonciation des «fausses dévotions » et des «faux dévots» lui attirait l'hostilité du «petit peuple $»^{24}$. Jean Richard pensait que le combat d'Érasme était à poursuivre en un temps où avaient encore cours «histoires ridicules» et «faux miracles $»^{25}$, où n'étaient pas efficacement réprimées les «propositions impies » des moralistes laxistes, et où au contraire « 5 propositions fabriquées à plaisir, qui ne se trouvaient dans aucun auteur $»^{26}$ étaient l'objet de toute la sévérité des autorités.

Cette défense d'Érasme, « le plus savant homme de son siècle », qui s'était attiré l'hostilité des «moines » et des «demi savants", le restaurateur des belles-lettres et le défenseur du catholicisme contre les protestants, était alors devenue lieu commun, aussi bien sous la plume de l'auteur de l'article «Érasme» du Dictionnaire de Moréri, que sous celle d'Ellies du Pin dans sa Nouvelle bibliothèque des auteurs ecclésiastiques ${ }^{27}$ et dans son Histoire de l'Église et des auteurs ecclésiastiques du seizième siècle ${ }^{28}$.

\section{RICHARD SIMON DEVANT ÉRASME INTERPRÈTE DE L'ÉCRITURE}

C'étaient ces lieux communs que Richard Simon allait remettre en cause grâce à son expérience de la critique du Nouveau Testament et de l'étude rigoureuse des textes patristiques. Les traces sont nombreuses de la lecture attentive et critique d'Érasme que l'érudit, exclu de l'Oratoire en 1681, avait déjà largement commencée avant 1671 et qu'il poursuivra jusqu'à la fin de ses jours. Ces traces ne sont nombreuses qu'à partir du moment où Simon, ayant publié, avec toutes les traverses que l'on connaît, son Histoire critique du Vieux Testament, s'attachera à la rédaction d'une histoire critique du Nouveau Testament, abordant un domaine où l'humaniste du $\mathrm{XVI}^{\mathrm{e}}$ siècle ne pouvait pas ne pas apparaître comme un précurseur.

22. Ibid., p. 52-53.

23. Ibid., p. 62.

24. Ibid., p. 195, 253.

25. Ibid., p. 253-254.

26. Ibid., p. 322.

27. 6 vol., Paris, 1686-1691.

28. 5 vol., Paris, 1701-1703, t. I, p. 144-146, 331-333. 
Dans une lettre du 15 juillet 1683, Richard Simon s'interroge sur la traduction latine de saint Jean Chrysostome par Érasme et juge cette dernière "pas digne d'un homme qui s'était acquis une si grande réputation $»^{29}$. La même année, le $1^{\text {er }}$ août, rappelant au même correspondant l'éloge fait par Bucer des Paraphrases d'Érasme sur les Évangiles, Simon souligne la distance de la doctrine de l'humaniste qui s'attachait aux Pères grecs par rapport à celle de Bucer et des protestants, jugement où la dimension théologique paraît aussi importante que le souci critique ${ }^{30}$; nous retrouverons ces deux aspects, critique et théologie, dans la plupart des jugements de Simon sur Érasme: préférence de Simon pour les Pères grecs par rapport aux Pères latins (avec les conséquences théologiques impliquées par cette préférence), louange d'un Érasme qui, lui aussi, avait réhabilité les Pères grecs, même s'il «n'était pas si savant dans la langue grecque qu'on le croit ordinairement $»^{31}$. Jugement nuancé, comme celui que porte Simon en 1687, dans une lettre à son neveu, sur le débat entre Érasme et Jules Scaliger à propos du cicéronianisme, occasion pour le critique de justifier l'invention linguistique dans le domaine théologique ${ }^{32}$.

Ce sont là remarques dispersées au hasard des lettres conservées. Les grandes critiques du Nouveau Testament, Histoire critique du texte du Nouveau Testament (Rotterdam, 1689), Histoire critique des versions du Nouveau Testament (Rotterdam, 1690) et Histoire critique des principaux commentateurs du Nouveau Testament (Rotterdam, 1693) vont donner lieu à un dialogue direct de Richard Simon avec l'œuvre d'Érasme, et dans le domaine où ce dernier avait acquis une particulière autorité. Ne reprenons que les grandes lignes de ce dialogue en en évaluant les conséquences dans le domaine de la critique et dans celui de la théologie.

29. Richard Simon, Lettres choisies, Rotterdam, 1702, p. 103.

30. Ibid., p. 124-125.

31. Lettre à $\mathrm{J}$ [ohn] $\mathrm{H}$ [ampden] du 2 novembre 1684 , ibid., p. 216, et p. 213 : «Les Pères grecs m'ont toujours paru être fort supérieurs aux Pères latins, surtout dans ce qui regarde l'interprétation des livres sacrés ».

32. Richard Simon, Lettres choisies, t. III, Rotterdam, 1705, p. 117-121; et voir p. 118: «La religion chrétienne a des mystères pour lesquels il a fallu nécessairement inventer de nouveaux termes ou donner de nouvelles significations à ceux qui étaient déjà en usage, ou enfin latiniser les mots grecs dont les Évangélistes et les Apôtres se sont servis dans le Nouveau Testament ». 
À propos de l'histoire du texte du Nouveau Testament, Richard Simon avait peu de raisons de se référer à Érasme dont les travaux de critique textuelle lui paraissaient peu concluants. Si l'humaniste s'était opposé à l'hypothèse d'un évangile de Matthieu rédigé en hébreu (entendons : en araméen), c'est qu'il «n'avait aucune connaissance de la langue hébraïque », et «les raisons qu'il produit sont si faibles qu'il se rend même quelquefois ridicule lorsqu'il veut parler en critique d'une matière qu'il ignorait entièrement »; il ne faisait sur ce point que suivre Ecolampade qui n'en savait « guère plus que lui $»^{33}$.

Le jugement est moins brutal à propos de l'auteur de l'Épître aux Hébreux : même si Richard Simon pense qu'Érasme a répondu de façon trop «générale » à ses détracteurs qui l'accusaient de douter de l'attribution de l'épître à saint Paul, il n'est pas éloigné de la position de l'humaniste lorsqu'il affirme qu' «il se trouve même encore aujourd'hui de savants critiques qui doutent que saint Paul en soit l'auteur bien qu'ils la reconnaissent pour divine et canonique », et Simon peut conclure en notant qu'à tenir cette position il n'y a pas «matière d'hérésie $»^{34}$.

Cependant c'est à propos de la traduction du Nouveau Testament, des Notes et des Paraphrases qu'Érasme y a jointes que les jugements de Simon sont les plus nombreux et les plus approfondis. En effet Érasme fut, selon l'ancien oratorien, «le premier qui ait composé dans ces derniers temps une version latine entière de tout le Nouveau Testament sur l'original grec $»^{35}$. Mais les éloges et les réserves exprimés à l'égard du travail d'Érasme vont alterner: sans cautionner les objections faites par les théologiens qui regardaient cette traduction «comme une nouveauté pernicieuse à l'Église principalement à cause des Notes qu'il y avait jointes $»^{36}$ et en rappelant comment la lettre de l'auteur à Léon $\mathrm{X}$ avait répondu à ces « demi-savants » qu'il « imitait [...] les théologiens du commun qui appuient sur l'autorité de l'Église tout ce qui est reçu par un long usage $»^{37}$, Simon reprochait à la

33. Richard Simon, Histoire critique du texte du Nouveau Testament, Rotterdam, 1689, p. 48.

34. Ibid., p. 171, 183.

35. Richard Simon, Histoire critique des versions du Nouveau Testament, Rotterdam, 1690, p. 242.

36. Ibid., p. 243.

37. Ibid., p. 244-245. 
critique exercée par Érasme d'avoir «été trop précipitée $»^{38}$ et de ne pas rendre justice à l'ancienne traduction, à la Vulgate, qui justement avait été corrigée par ordre des papes et par des théologiens qui n'étaient pas «du commun $»^{39}$. Quel était alors aux yeux de Simon le défaut essentiel de la traduction d'Érasme? C'était paradoxalement d'être tombé lui-même dans le travers dont il accusait les autres: "Je veux bien croire que ceux qui ont condamné la version d'Érasme et ses Notes n'avaient pas une connaissance exacte de la matière qu'ils traitaient, et que c'est ce qui les a obligés à se jeter sur des lieux communs ou sur des questions générales de théologie. Mais il avait lui-même donné occasion à cela parce qu'il fait quelquefois le théologien dans ses Notes $»^{40}$. D'où le tableau de ce qu'aurait dû faire Érasme : ne pas « refondre entièrement une version », mais corriger " par de petites notes » les «prétendus défauts » de l'ancienne, de la Vulgate ${ }^{41}$.

Car Érasme ne s'était pas donné, ou n'avait pu avoir de son temps, les moyens de réaliser une version à la fois neuve et satisfaisante aux yeux de Simon. Certes il avait eu recours à quelques manuscrits grecs du Nouveau Testament, « et il mérite de grandes louanges pour la recherche qu'il en a faite dans un temps que la critique n'était guère du goût des théologiens $»^{42}$, mais il a eu trop de confiance dans les quelques manuscrits qu'il a vus.

Quelles conclusions en tirer, et quelle utilité peut-on trouver à cette version? Car l'utilité, dans le sens le plus large, englobant aussi bien la critique que la théologie, est une notion centrale dans la pensée et la pratique de Richard Simon. Si ce dernier critique Érasme pour les nouveautés injustifiées qu'il a introduites dans sa traduction, ce n'est pas par conservatisme, c'est que, contrairement à ce qu'on pourrait croire, le critique manifeste toujours, à côté des exigences «scientifiques» de critique textuelle proprement dite, un sens très aigu de la tradition, de ce qu'on pourrait appeler la dimension sociale d'un texte, de sa production et de ses usages.

Un usage crée, à ses yeux une légitimité, une «canonicité », des notes pouvant à l'occasion préciser l'écart entre l'expression

38. Ibid., p. 247.

39. Ibid., p. 246.

40. Ibid., p. 248.

41. Ibid., p. 249.

42. Ibid., p. 250. 
usuelle et le sens littéral du texte. "Il y a, écrit Simon, dans notre version latine de certains mots qu'on peut appeler des mots consacrés parce qu'ils sont reçus depuis plusieurs siècles dans les Églises d'Occident. Érasme a été plus exact à les conserver que la plupart des autres traducteurs. [...] J'aurais même souhaité qu'il eût gardé plus d'uniformité qu'il n'a fait à l'égard de ces mots consacrés et de quelques autres. [...] On ne doit point changer l'ancienne façon de parler qui est reçue depuis longtemps dans l'Église $»^{43}$. Même un protestant comme Jean Boys, écrit Simon, «improuve ceux qui ont changé sans aucune nécessité des mots autorisés par un long usage $»^{44}$. Et Simon donne quelques exemples de ces inutiles changements opérés par Érasme: le changement de verbum en sermo au premier verset du premier chapitre de l'évangile de Jean, ou, au verset 41 du même chapitre, celui de christus en unctus; même si l'exactitude littérale justifie Érasme, il vaut mieux garder christus «parce que l'usage de l'Église a canonisé christus $»^{45}$.

Nous voyons ainsi quelle est la double et inséparable préoccupation de Simon: à la fois la recherche de l'exactitude littérale obtenue par le recours aux manuscrits et par une rigoureuse critique textuelle, et la considération de l' « usage » dans la société qu'est l'Église catholique, usage qui «autorise» des expressions et qui donne un statut «canonique », qui « canonise » tel vocable. D'où le caractère que l'on pourrait appeler «tridentin» de la critique simonienne, le concile de Trente ayant «canonisé » la Vulgate, se contentant de recommander des corrections sur tel ou tel point ${ }^{46}$. Ainsi Érasme peut apparaître comme «le plus exact de tous les nouveaux traducteurs du Nouveau Testament », mais «il n'a pas eu raison de s'éloigner en beaucoup d'[...] endroits de l'ancien interprète latin $\gg^{47}$, et cela d'autant plus que les

43. Ibid., p. 255.

44. Ibid., p. 256.

45. Ibid., p. 258 ; de même Simon note, p. 259, que «le mot de Paracletus est en quelque façon un terme canonisé ». Sur le souci d'Érasme, que malgré ses critiques reconnaît Simon, de conserver les vocables traditionnels, voir Henri de Lubac, Exégèse médiévale, op. cit., II' partie, t.2, p. 427-428.

46. Sur cet aspect de l'œuvre de Richard Simon, voir Jacques Le Brun, La jouissance et le trouble, Genève, Droz, 2004, p. 195-216.

47. R. Simon, Histoire critique des versions du Nouveau Testament, op. cit., p. 262. 
conséquences théologiques de cet écart par rapport à la tradition peuvent être considérables. L'exemple le meilleur serait ici la modification apportée par Érasme dans la traduction de Romains 5, 12, l'humaniste traduisant par quatenus omnes peccaverunt là où la Vulgate donnait in quo omnes peccaverunt; toute une théologie de la chute, de la grâce et de la rédemption est en cause dans ces deux traductions.

Quelle sera alors la tâche du critique selon Simon? À quelle exigence, critique ou théologie, doit-il donner la priorité ? «Il eût été mieux, écrit Simon d'Érasme, [...] de ne point abandonner l'ancien interprète de l'Église dans un endroit de cette importance. Il devait réserver pour ses Remarques ce qu'il a mis dans sa version $»^{48}$.

Nous comprenons que, dans ces conditions, Richard Simon ait consacré deux gros chapitres de son Histoire critique des principaux commentateurs du Nouveau Testament ${ }^{49}$ aux Notes jointes par Érasme à sa traduction et aux Paraphrases qu'il a composées du Nouveau Testament. Certes Érasme a joint à sa traduction de petites «notes », annotatiunculas, qui donnent «les véritables leçons du texte $\gg^{50}$; s'il s'en était tenu là, Simon ne pourrait que l'approuver, mais Érasme a fait le « déclamateur», a multiplié les « digressions tout à fait inutiles et hors de propos », ce qui n'est pas le fait d' « un critique exact». Vengeance contre les moines, «fausses plaisanteries $»^{51}$, «questions hors de propos $»^{52}$ envahissent ainsi les notes. De plus Érasme «fait le théologien », 《s'érige en canoniste $»^{53}$, se jette dans la «théologie», cède à la tentation de « dogmatiser ${ }^{54}$, ce n'était là ni la tâche d'un critique, ni celle d'un interprète ou d'un paraphraste.

Peut-on néanmoins se fier à ses talents de grammairien et de critique? En réalité Érasme ignorait l'hébreu - Simon l'avait déjà souligné dans son précédent ouvrage - et il s'appuyait sur Ecolampade «qui n'avait presque aucune connaissance des faits dont il s'agissait », il voulait « réformer saint Jérôme quand

48. Ibid., p. 262.

49. Richard Simon, Histoire critique des principaux commentateurs du Nouveau Testament, Rotterdam, 1693, p. 504-536.

50. Ibid., p. 504.

51. Ibid., p. 505-506.

52. Ibid., p. 509.

53. Ibid., p. 515.

54. Ibid., p. 517, 518. 
il ne l'entend point», il jugeait «avec trop de précipitation de quelques exemplaires grecs », ses citations «ne sont pas toujours exactes », il tombe "dans quelques erreurs en ce qui regarde le sens grammatical et littéral $»^{55}$, il n'a pas apporté à ses paraphrases l'attention qu'elles méritaient, en un mot, comme sans le nommer écrivait Latomus, «il parlait en demi-savant de certaines choses » et sa science est souvent affectation ${ }^{56}$.

Érasme a donc ignoré «les véritables règles de la critique $»^{57}$ qui inspirent le travail de Richard Simon. Et pourtant, malgré toutes ces réserves, le jugement que selon Simon on doit porter sur l'œuvre d'Érasme n'est pas tout négatif ; l'humaniste a dissipé des 《 préjugés » reposant sur des «étymologies absurdes $»^{58}$, on peut le juger «un des plus habiles critiques de son temps $»^{59}$, et ses paraphrases «méritent d'être lues, parce qu'il y éclaircit plusieurs faits de critique et de théologie avec beaucoup d'érudition $»^{60}$.

Par ailleurs Simon juge avec nuances la théologie d'Érasme : certes il lui reproche d'avoir quitté le terrain de la critique pour celui de la théologie, et cette théologie elle-même n'est pas exempte de suspicion. Si on peut justifier Érasme du reproche de pélagianisme - et Simon, prêt à le justifier sur ce point, cite avec bienveillance les critiques portées par lui contre saint Augustin $^{61}$ il n'en va pas de même pour une certaine tendance à l'arianisme que manifestent plusieurs remarques d'Érasme qui détourne en faveur des ariens certains passages du Nouveau Testament ${ }^{62}$.

55. Ibid., p. 506-510.

56. Ibid., p. 526-527.

57. Richard Simon, Dissertation critique à la suite de l'Histoire critique des principaux commentateurs du Nouveau Testament, op. cit., p. 6.

58. Richard Simon, Histoire critique des principaux commentateurs du Nouveau Testament, op. cit., p. 506.

59. Ibid., p. 516.

60. Ibid., p. 521.

61. Ibid., p. 530 .

62. Ainsi Romains 9, 5. Sur les accusations d'arianisme lancées de longue date contre Érasme, voir Marcel Bataillon, Érasme et l'Espagne, rééd. Genève, Droz, 1998, en particulier p. 267-268. Sur la reprise par Grotius de ces orientations favorables à l'arianisme, voir Jacques Le Brun, La jouissance et le trouble, op. cit., p. 236-237. 


\section{DEVANT LES PRINCIPES THÉOLOGIQUES D’ÉRASME}

Avec la lucidité qui le caractérise, Richard Simon avait bien repéré ce qui était essentiel dans la distance qui séparait de l'érudit du $\mathrm{XVI}^{\mathrm{e}}$ siècle un critique de la fin du XVII ${ }^{\mathrm{e}}$. Il ne s'agissait pas d'un simple accroissement des connaissances, au demeurant incontestable ${ }^{63}$, mais d'une autre façon de faire de la critique textuelle ${ }^{64}$, et d'une autre façon de la théologie par un nouveau rapport aux sources et un nouvel usage de ces sources. «Il devait avoir appris, écrit Simon d'Érasme, que depuis que la théologie avait été réduite en art par les docteurs scolastiques, il fallait se soumettre à de certaines règles et à de certaines manières de parler; qu'il ne s'agissait plus de savoir ce qu'on lisait dans les anciens écrivains ecclésiastiques puisqu'il demeurait lui-même d'accord qu'ils ne convenaient point entr'eux, outre qu'il n'avait produit dans ses Notes que de simples extraits de leurs ouvrages, qui ne découvraient pas toujours leurs véritables pensées $»^{65}$.

Ainsi la critique de Richard Simon, héritant de la révolution cartésienne qui s'était acclimatée à l'Oratoire ${ }^{66}$, est caractérisée par une double mutation : dans l'approche, l'établissement et l'analyse des textes, et dans la conception même du travail théologique et de ce qui fait « autorité », ce qui « autorise » dans le champ de la théologie. Les deux aspects sont étroitement liés. Aussi, lorsque Richard Simon analyse dans sa Bibliothèque critique ${ }^{67}$ les livres de Noël Beda contre Le Fèvre d'Étaples et contre Érasme, il peut relever le mépris des « savants dans les humanités » à l'égard des «professeurs de théologie», mais c'est pour renvoyer pour ainsi dire dos à dos les uns et les autres, les docteurs négligeant trop «l'étude de l'Écriture et de l'ancienne théologie», les savants

63. Sur l'apport essentiel de Simon dans la critique du Nouveau Testament voir Leighton Durham Reynolds et Nigel Guy Wilson, D'Homère à Érasme, nouv. éd. revue et augmentée, Paris, C.N.R.S., 1988, p. 128-129.

64. «Les véritables règles de la critique » dont Simon revendique l'usage et qu'à son époque Jean Le Clerc codifiait dans son Ars critica.

65. Richard Simon, Histoire critique des principaux commentateurs du Nouveau Testament, op. cit., p. 535.

66. Voir Jacques Le Brun, «Vérité scientifique et vérité historique à l'âge classique », La vérité entre psychanalyse et philosophie, Michel Plon et Henri Rey-Flaud éd., Ramonville-Saint-Agne, Érès, 2007, p. 45-70.

67. Amsterdam, t. II, 1708, p. 376 et suiv. 
méprisant les docteurs sous prétexte qu'ils n'avaient aucune connaissance des belles-lettres ${ }^{68}$.

Tous ces arguments seront repris par Richard Simon dans les chapitres de sa posthume Critique de la Bibliothèque des auteurs ecclésiastiques $^{69}$. On y retrouve les nuances des jugements que de longue date Simon avait portés sur l'humaniste; on y trouve un éloge de celui qui avait «déterré » et «donné au public» les Notes de Lorenzo Valla sur le Nouveau Testament ${ }^{70}$, mais aussi de graves réserves sur la science d'Érasme: ce dernier n'aurait nullement été « le plus savant homme de son siècle » ${ }^{71}$, il y avait eu bien de savants critiques en Italie et « il n'était nullement exercé dans la science de l'antiquité ecclésiastique $»^{72}$. Par ailleurs il ne « savait que médiocrement» la langue grecque ${ }^{73}$ et absolument pas l'hébreu ${ }^{74}$. Richard Simon souligne aussi l'infériorité d'Érasme en matière de théologie ${ }^{75}$.

Ces jugements ne sont-ils pas en contradiction avec ceux que formulait autrefois Richard Simon? Si on lit le chapitre consacré à Vivès dans la Critique de la Bibliothèque des auteurs ecclésiastiques $^{76}$, on y trouve une violente diatribe qui rompt avec le ton mesuré des jugements publiés jadis dans les Critiques du Nouveau Testament : «faux brillant propre à faire illusion à bien des gens », « déclamateur », « sophiste », « invectives », « fécond en paroles », « abondance de mots $»^{77}$. En contraste, nous lisons un vif éloge des livres de Louis Vivès : "Il y a plus à profiter pour la science des arts dans les petits livres de Vivès que dans tous

68. Ibid., p. 381.

69. Paris, 1730. Au tome I, on consultera les chapitres sur Valla, sur Érasme, sur Latomus, sur Ed. Lee, sur Stunica, sur Vivès. Sur ces auteurs, voir évidemment Marcel Bataillon, Érasme et l'Espagne, op. cit.

70. Richard Simon, Critique de la Bibliothèque..., op. cit., t. I, p. 385.

71. Ibid., t. I, p. 583.

72. Ibid., t. I, p. 583-584.

73. Ibid., t. I, p. 585, à partir du jugement porté par Castellan d'après la vie de ce dernier par Pierre Galland publiée par Baluze. C'était d'ailleurs le jugement de Bayle qui s'appuyait sur le même témoignage : voir l'article «Érasme » du Dictionnaire historique et critique. Dans sa lettre du 15 juillet 1683, Simon rapportait déjà, à partir de Castellan, la faiblesse de la connaissance du grec par Érasme, Lettres choisies, Rotterdam, 1702, t. I, p. 106-107.

74. Richard Simon, Critique de la Bibliothèque..., op. cit., t. I, p. 594, 599, 604.

75. Ibid., t. I, p. 592, 596-597, 603.

76. Ibid., t. I, p. 611-638.

77. Ibid., t. I, p. 635. 
les livres d'Érasme qui sont à la vérité plus à la portée des jeunes gens qui étudient la langue latine, mais les savants et les personnes judicieuses n'ont pas ce même goût pour les livres d'Érasme. S. Ignace et ses disciples n'ont pu les souffrir $\gg^{78}$. À supposer que ces pages soient bien de Richard Simon - et ces textes publiés par le P. Souciet, s. j., après la mort de Simon peuvent avoir été, sinon remaniés, du moins sélectionnés dans des intentions précises - nous aurions là un jugement beaucoup moins favorable que ceux qui avaient été publiés auparavant. Ultime pensée de Simon ? Ou réaction d'humeur du critique devant les éloges peu nuancés formulés par Moréri, par Baillet, ou par Ellies du Pin $^{79}$, en attendant ceux de Jacques Marsollier qui, en 1713, dans son Apologie ou justification d'Érasme ${ }^{80}$, faisait de son héros celui qui avait su dénoncer nombre de pièces fausses ou supposées, la Légende dorée de Voragine ou les Légendes de Métaphraste ${ }^{81}$ et qui « donna des règles pour discerner le vrai d'avec le faux et [qui] fut l'un des premiers qui fit un art de la critique et qui apprit à juger sainement de toutes choses $»^{82}$, enfin celui qui a «traité les matières de théologie d'une manière noble et dégagée des sophistiqueries et des termes de l'École ${ }^{83}$, bref, aux yeux de Marsollier, un parfait savant « éclairé »!

Richard Simon apparaît plus proche de la position d'un Le Clerc qui, dans la préface de sa grande édition des Opera omnia d'Érasme, publiée à Leyde en 1703, reconnaissait les limites de l'érudition de son auteur ${ }^{84}$, mais estimait qu'il avait puisé la

78. Ibid., t. I, p. 636. Sur l'attitude des jésuites devant Érasme voir les remarques de Jean-Claude Margolin, «La fortune d'Érasme : l'exemple anglais », Les Jésuites à l'âge baroque, Luce Giard et Louis de Vaucelles éd., Grenoble, Jérôme Millon, 1996, p. 79-88.

79. Encore un peu plus tard Honoré de Sainte-Marie, Réflexions sur les règles et l'usage de la critique touchant l'histoire de l'Église..., t. I, Paris, 1718, p. 45 n. : «On donne la gloire à Érasme d'avoir été le premier des savants qui ait tenté la critique des ouvrages des Pères de l'Église. Il a corrigé leurs exemplaires d'une infinité de fautes »; mais quelques réserves suivent : «On a grand sujet de s'en défier».

80. Paris, 1713. Les jésuites en feront la critique dans les Mémoires de Trévoux en mars 1714.

81. Jacques Marsollier, Apologie..., op. cit., p. 167.

82. Ibid., p. 168.

83. Ibid., p. 242.

84. Érasme, Opera omnia, t. I, Leyde, 1703, Préface, p. 2 : «Saepe erravit, pravis malarum editionum lectionibus deceptus ; interdum etiam dormitavit...», p. $6:$ : hominem [...] eum puto fuisse, non Deum». 
théologie chrétienne aux sources qu'il jugeait les plus limpides ${ }^{85}$. Pour Le Clerc, comme pour Richard Simon, Érasme devait être jugé « eu égard au temps qu'il écrivait ${ }^{86}$. Après plus d'un siècle de travaux érudits, après le tournant tridentin du catholicisme et après l'affirmation de la méthode cartésienne, Richard Simon, comme Jean Le Clerc, était un témoin et un acteur des mutations de la critique. L'œuvre d'Érasme prenait place dans une histoire, un moment du passé ; la position même du critique avait changé, sa position devant la «vérité » des textes, sa position devant les institutions ecclésiastiques.

Ainsi se comprennent les paradoxes et les apparentes contradictions de l'attitude de Richard Simon vis-à-vis d'Érasme : ces paradoxes et ces contradictions peuvent apparaître comme inséparables de l'attitude du critique à l'égard des documents sur lesquels se fonde le christianisme. Érasme affectait de ne pas juger l'œuvre de saint Augustin et de ne l'aborder qu'en grammairien, mais c'était pour s'opposer à la théologie et à l'anthropologie augustiniennes; contre saint Augustin il exaltait saint Jérôme et voyait en ce dernier le modèle du philosophe chrétien et le seul théologien digne de ce nom ${ }^{87}$. Un siècle et demi plus tard, Richard Simon, avec d'autres instruments scientifiques et tout en critiquant les lacunes de la science d'Érasme, reprenait le flambeau, exaltant saint Jérôme, moyen sinon d'attaquer ouvertement, du moins de saper l'autorité de saint Augustin en théologie. Le critique affectait de se tenir à l'écart du débat proprement théologique, mais c'était peut-être pour mieux mettre au jour les faiblesses de la théologie des Écoles et pour faire de la critique textuelle et de la critique historique les conditions incontournables de toute élaboration théologique. Que l'un et l'autre, Érasme et Richard Simon, aient largement échoué du point de vue des institutions ecclésiastiques n'empêche pas la question de ressurgir à chaque génération. L'interminable et insoluble crise moderniste au $\mathrm{Xx}^{\mathrm{e}}$ siècle témoigne

85. Ibid., p. 3.

86. R. Simon, Histoire critique des principaux commentateurs..., op. cit., p. 518.

87. Voir Viviane Mellinghoff-Bourgerie, «Érasme éditeur et interprète de saint Augustin ", Augustinus in der Neuzeit, Kurt Flasch et Dominique de Courcelles éd., Turnhout, Brepols, 1998, p. 53-81. Et voir notre article «L'antiaugustinisme de Richard Simon », Revue de l'histoire des religions, avril-juin 2012, p. 257-273. 
sans doute de la pérennité de cette question fondamentale. Ce ne sera pas un hasard si en 1900, avant de quitter la prêtrise l'année suivante, l'abbé Henri Margival, proche du modernisme, publie un Essai sur Richard Simon et la critique biblique au XVII siècle, que Loisy dans ses Mémoires qualifiait de « remarquable ${ }^{88}$.

jacleb@ orange.fr

88. Alfred Loisy, Mémoires pour servir à l'histoire religieuse de notre temps, t. I, Paris, Nourry, 1930, p. 397. 